\title{
Novel Detection System for Calcium(II) Ions Based on an Apoenzyme Reactivation Method Using an Amylase Column as a Recognition Element
}

\author{
Yasuhiro IIDA, Yumiko SATO, and Ikuo SATOH*
}

\begin{abstract}
Department of Applied Chemistry, Faculty of Engineering, Kanagawa Institute of Technology (1030 Shimo-Ogino, Atsugi, Kanagawa-ken 243-0292, Japan)
\end{abstract}

Received December 9, 2002 ; Accepted March 4, 2003

\begin{abstract}
A flow-injection amperometric system for evaluation of the activity of an immobilized $\alpha$-amylase column was constructed and the system was applied to detect of calcium(II) ions based on the apoenzyme reactivation method. The $\alpha$-amylase activity was amperometrically evaluated by using a double-layered column which consisted of $\alpha$ glucosidase and pyranose oxidase. The detection of calcium(II) ions, being the cofactor of $\alpha$-amylase, was realized by measuring the reactivation of apo-type $\alpha$-amylase column, which was the recognition element of $\mathrm{Ca}$ (II) ions. Maleate buffer $(0.1 \mathrm{M}, \mathrm{pH} 6.0)$ as a carrier solution was continuously pumped through the system, and sample solutions were introduced into the system via rotary injection valves. The catalytic activity of the multi-enzyme column was assessed by injecting $0.5 \%$ starch solution as a substrate. Calcium(II) ions in $1.0 \mathrm{mM}$ levels were detected by using the system based on the apoenzyme reactivation method.
\end{abstract}

Key Words : $\alpha$-Amylase, Apoenzyme Reactivation Method, Calcium, FIA, Biosensor

\section{Introduction}

We investigated the apoenzyme reactivation methods for flow-injection analysis (FIA) of heavy metal ions. ${ }^{1-31}$ The methods provide the assay for heavy metals by measuring the reactivation ratio of the column packed with immobilized apoenzymes, i.e., cofactor-free enzymes from metalloenzymes. The apoenzymes are regenerated by removing the metal ions located in the active site of the metalloenzymes, and the enzyme-column can be reactivated. In our first report on the apoenzyme reactivation method, a carbonic anhydrase-immobilized column was used and the determination range of zinc(II) ions was $0.025-0.25 \mathrm{mM}^{4}{ }^{4)}$ Lately, several kinds of metalloenzymes have been examined as the recognition elements for divalent metal cations such as cobalt(II), copper(II) and zinc(II) ions ${ }^{1-3)}$ and it was found that these metal ions in ultratrace levels have been successfully determined. ${ }^{5,6)}$ The apoenzyme reactivation methods possess unique properties which can offer nonhazardous, sensitive, mild and selective assay. Furthermore, as the sensing system has quite a simple construction, the system can be easily miniaturized and provide the cost-effective method in comparison with conventional methods such as atomic absorption spectrometry, and inductively coupled plasma (ICP) emission spectrometry. Thus, the proposed methods are promising for monitoring heavy metal ions in the analytical fields such as environmental assessment, clinical analysis and quality control in food industry. From the view point of another potential of the apoenzyme reactivation method, this method is also promising to be applicable to recognition element for determination of many other metal ions using various met- alloenzymes. There are so many kinds of metalloenzymes, and many kinds of metal ions play roles as cofactors for the corresponding metalloenzymes. It is known that calcium(II) ions are the cofactor of $\alpha$-amylase, ${ }^{7}$ and the apoenzyme reactivation method using $\alpha$-amylase will provide a novel determination method for calcium(II) ions. It is widely known that the calcium has divergent roles in human body such as the main ingredients of exo and endoskeleton, muscular contraction, neurotransmission, coagulation and so on. It is also well known that the concentration of calcium in blood is severely regulated by parathyroid hormone at about $2.0 \sim 2.5 \mathrm{mM}$, and abnormal level in the calcium indicates the probability of being serious diseases. Therefore, analysis of calcium in blood is important. In this study, to measure the calcium (II) concentration, we have constructed a system for measurement of $\alpha$-amylase activity using a multi-enzyme column FIA system. Then, we applied the FLA system in combination with the apoenzyme reactivation method using the column as a recognition element for calcium determination.

\section{1 Materials}

\section{Experimental}

$\alpha$-Amylase (E.C. 3.2.1.1, from Bacillus subtillis) was generously donated by Daiwa Kasei Co., Ltd. (Osaka, Japan). $\alpha$-Glucosidase (E.C. 3.2.1.2, from brewers yeast) and pyranose oxidase (E.C. 1.1.3.10, from Coriolus versicolor) were obtained from Sigma Chem. Co. and Kyowa Medex Co. Ltd. (Tokyo), respectively. Controlled-Pore Glass (CPG, mean pore diameter $24.2 \mathrm{~nm}$, particle size 120-200 mesh) was purchased from Funakoshi Co., Ltd. 


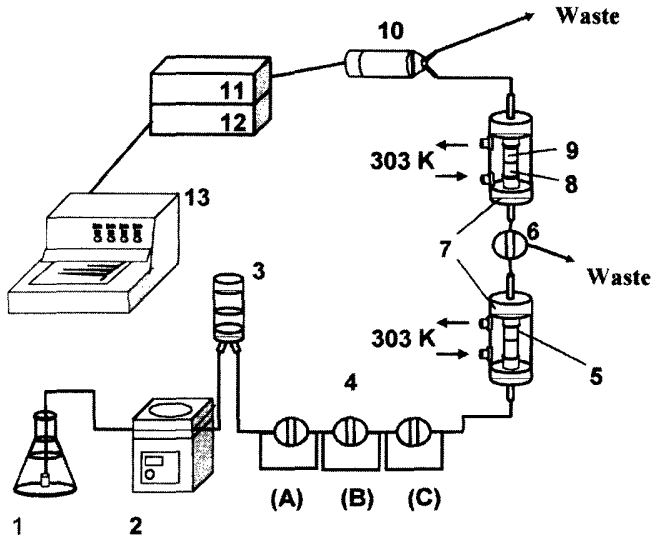

Fig. 1 Schematic drawing of the amperometric FIA system using the multiple enzyme columns. 1) Carrier reservoir, 2) Peristaltic pump, 3) Pulse damper, 4) Injection valves: (A) for chelating agent, (B) for standard calcium(II) ions, (C) for substrate, 5) $\alpha$-amylase column $(0.3 \mathrm{ml}), 6)$ Switching valve, 7) Water jackets, 8) $\alpha$-glucosidase column $(0.15 \mathrm{ml})$, 9) Pyranose oxidase column $(0.15 \mathrm{ml}), 10) \mathrm{O}_{2}$ electrode, 11) Potentio-galvanostat, 12) Electrometer, 13) Pen recorder.

(Tokyo). Starch as substrates for amylase and the standard calcium solution (Ca 1000, atomic absorption spectrometry grade) as a cofactor of the enzyme were purchased from Merck (Darmstadt, Germany), and all other reagents were commercially available in analytical grades and were used without further purification.

\section{2 Flow system and procedure}

Three enzymes were covalently immobilized onto alkylaminated $\mathrm{CPG}$, respectively, and their preparation method was previously described. ${ }^{8}$ The immobilized amylase was packed into a small polymer column and $\alpha$ glucosidase and pyranose oxidase were double-layered into the other small polymer column, where each enzyme layer was separated with a Vyon (polyethylene) filter. Both columns, amylase ( $0.3 \mathrm{ml}$ packed volume) and double-layered enzymes (packed volume of each enzymes was $0.15 \mathrm{ml}$ ), were mounted in a water-jacketed holder regulated at $303 \pm 0.1 \mathrm{~K}$. The enzyme columns were used as the recognition elements for $\alpha$-amylase. A schematic diagram of the flow system was shown in Fig. 1.

Maleate buffer solution (100 mM, pH 6.0) as the carrier was continuously pumped through the system. The sample solutions were introduced into the system through the rotary injection valve. The catalytic activities of the enzyme-packed column were assessed by injecting $0.1 \mathrm{ml}$ of starch solutions with various concentrations. For preparation of the substrate of amylase, starch was dissolved in $100 \mathrm{mM}$ maleate buffer, which was used as a carrier solution by heating in a microwave oven. Variation in the dissolved oxygen caused by the enzymecatalyzed reaction was monitored by the flow-through type of a polarographic oxygen electrode (BO-P, ABLE Corporation, Tokyo, Japan) and then recorded.

2. 3 Calcium detection by the apoenzyme reactivation method

The application of the apoenzyme reactivation method in combination with $\alpha$-amylase column to determination of calcium(II) ions was investigated. The amylase activity was assessed by injecting $0.1 \mathrm{ml}$ of $0.5 \%$ starch solution as a substrate, and the change in the concentration of dissolved oxygen due to the enzyme catalysis was detected with the oxygen electrode. After the switching valve 6 was switched over waste, EDTA solution as a chelating agent was injected into the FIA system for removal of calcium(II) ions from the active site of the immobilized $\alpha$-amylase. Sample solutions and standard calcium solutions in this study were introduced into the system for the reactivation of the chelator-exposed enzymes. After switching back the valve 6 to return back, the amylase activity was evaluated by introducing the starch solution. Since the reactivation of the immobilized $\alpha$-amylase column depends on the amount of calcium ions added, the calcium existence in the analyte was detected by the measurement of the reactivation ratio of the $\alpha$-amylase column.

\section{Results and Discussion}

\section{1 Measurements of the response of the FIA sys-} tem

The starch solutions were hydrolyzed by three kinds of enzymes as follows:

$$
\begin{aligned}
& \text { Starch }+\mathrm{n} \mathrm{H}_{2} \mathrm{O} \text {-Amylase } \\
& \text { three or more } 1,4-\alpha \text {-linked D-glucose units } \\
& \text { Maltose and more } 1,4-\alpha \text {-linked } \alpha \text {-(ilucosidase } \\
& \text { D-glucose units }+\mathrm{n} \mathrm{H}_{2} \mathrm{O} \stackrel{\mathrm{n} \alpha \text {-D-glucose }}{\longrightarrow} \\
& \text { Pyranose oxidase } \\
& \alpha \text {-D-glucose }+\mathrm{O}_{2} \longrightarrow 2 \text {-dehydro-D-glucose }+\mathrm{H}_{2} \mathrm{O}_{2}
\end{aligned}
$$

Above the reactions, pyranose oxidase (PROD) is one of the oxidases, and therefore its activity can be evaluated by measuring the dissolved oxygen which is consumed in the catalysis of PROD. The activity of PROD depended on the amount of D-glucose which was produced by the other two enzymes hydrolysis. Therefore, the activity of $\alpha$-amylase could be evaluated by measuring the change in amount of dissolved oxygen due to PROD catalysis.

One milliliter of $1.0,0.5$ and $0.25 \%$ starch solutions was separately injected into the sensing system, and the $\alpha$-amylase activity was amperometrically monitored using the oxygen electrode. Figure 2 indicates the responses of $\alpha$-amylase to starch using the multi-enzyme FIA system. Figure 2 shows that the response currents of the oxygen electrode become higher as the concentration of starch is increased. The relative standard deviations of the samples of these three concentrations were below 1.5\% $(n=5)$. The output current of the oxygen electrode used in this study was usually about 3.6-3.7 $\mu \mathrm{A}$. Therefore, the response current obtained by injecting $1.0 \%$ starch solution indicates that the maximal amount of dissolved oxygen was consumed by the enzymecatalyzed reaction. In addition, the assay for $\alpha$-amylase activity by introducing $1.0 \%$ of starch solution took much more time in comparison with that of $0.5 \%$ injection. Therefore, $0.5 \%$ starch solution was employed for 


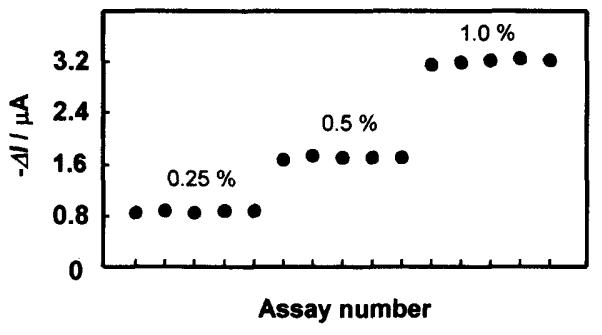

Fig. 2 Responses of the $\alpha$-amylase column to starch solution detected by the multiple enzyme columns. The $0.1 \mathrm{ml}$ of $0.25,0.5,1.0 \%$ starch solutions were injected into the FIA system. $\Delta I$ indicates the value of current response changed with constant-potential amperometry at $-700 \mathrm{mV}$ versus $\mathrm{Ag}$ / $\mathrm{AgCl}$.

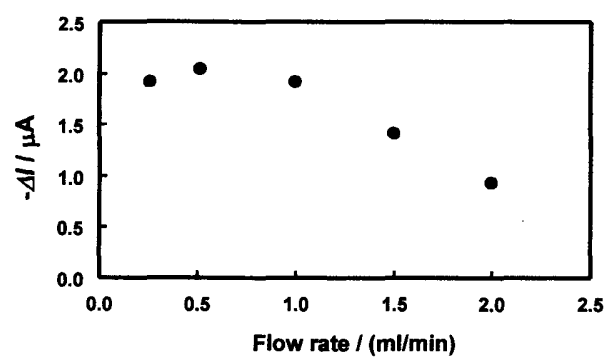

Fig. 3 Effect of the flow rate on the FIA system.

the evaluation of the $\alpha$-amylase activity in subsequent experiments.

3. 2 Effect of flow rate on the response of the FIA system.

The flow rate of the carrier solution was an important factor for regulating assay frequency. The increase in the flow rate shortened the assay time. On the other hand, the decrease in the flow rate provided sufficient time for completing the assay and, thereby, it brought a high sensitivity. However, if the flow rate were to be too slow, the sensitivity would be lowered due to the diffusion of the analyte toward surroundings. Therefore, the optimal flow rate was investigated. Figure 3 shows the effect of the flow rate on the response of the amperometric FIA system. The starch solution $(0.5 \%, 0.1 \mathrm{ml})$ was injected into the system, of which flow rates ranged from 0.25 to 2.0 $\mathrm{ml} / \mathrm{min}$. In proportion to the increase in the flow rate, the response of the system decreased except for the flow rate of $1.0 \mathrm{ml} / \mathrm{min}$. When the rate was $0.5 \mathrm{ml} / \mathrm{min}$, the multi-immobilized columns exhibited the maximal activity. However, the response of the $\alpha$-amylase column at 0.5 $\mathrm{ml} / \mathrm{min}$ was not so much different from the response of the column at $1.0 \mathrm{ml} / \mathrm{min}$. Therefore, it was considered that the optimal flow rate should be $1.0 \mathrm{ml} / \mathrm{min}$ in which a high sensitivity and a short operating time were obtained.

3. 3 Influence of chelating agents on $\alpha$-amylase activity.

The apoenzyme reactivation method essentially needs a removal of the cofactor from the metalloezyme. Therefore, the enzyme columns were exposed to several chelating agents such as EDTA, EGTA $\left(O, O^{\prime}\right.$-bis (2aminoethyl)-ethyleneglycol- $N, N, N^{\prime}, N^{\prime}$-tetraacetic acid)

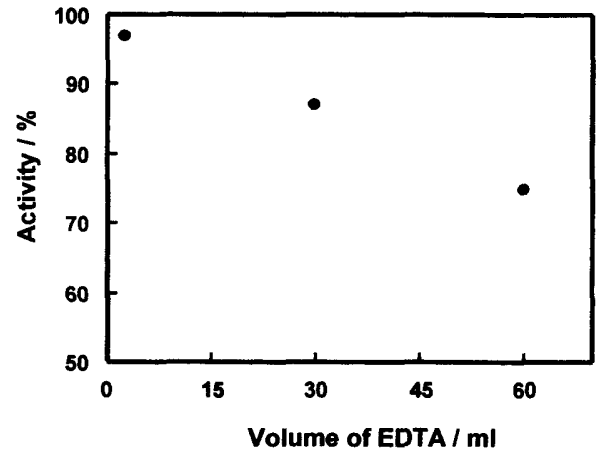

Fig. 4 Influence of chelating agents on the $\alpha$-amylase column.

and PDC (2,6-pyridinedicarboxylic acid) to remove the calcium(II) ions from the immobilized $\alpha$-amylase column. One milliliter of $100 \mathrm{mM}$ chelating agents was separately introduced into the FIA system. The injection of the EDTA and EGTA solutions reduced the activity of $\alpha$ amylase. PDC did not influence the activity of $\alpha$-amylase column (data were not shown). EDTA acts more effectively than EGTA on the $\alpha$-amylase activity, and then the influence of EDTA introduced on the $\alpha$-amylase activity was examined. Figure 4 shows the response attributable to the activity of $\alpha$-amylase column which was evaluated by the multi-enzyme column system followed by the treatment of $100 \mathrm{mM}$ EDTA solutions with various injection volumes. As the injection volume of EDTA solution increased, the activity of $\alpha$-amylase was decreased. When the column was exposed to $60 \mathrm{ml}$ of $100 \mathrm{mM}$ EDTA, the column activity was decreased to $75 \%$.

3. 4 Application of the apoenzyme reactivation method to the $\alpha$-amylase column for detection of calcium(II) ions.

The apoenzyme reactivation method was applied to $\alpha$ amylase column for detection of calcium(II) ions. This method was based on the reactivation ratio of regenerated enzyme, i.e., apoenzyme, to holoenzyme for the determination of its cofactor. The reactivation ratio was determined by calculating the differences between the response of holoenzyme and that of reactivated enzyme, which were divided by the differences between the response of holoenzyme and that of chelator-exposed enzyme. Each value was measured by injecting $0.1 \mathrm{ml}$ of $0.5 \%$ starch solution into the multi-enzyme columns using the oxygen electrode as a detector. As shown in Fig. $5,0.5 \mathrm{ml}$ of $100,10,1.0 \mathrm{mM}$ calcium standard solutions reactivate the apoenzyme column. The reactivation ratio was calculated to be $100,50,28 \%$, respectively. It indicates that the calcium(II) ions in the concentration of 1.0 $\mathrm{mM}$ were sufficiently detectable using the FIA system.

\section{Conclusion}

In this study, the FIA system attached to the multienzymes columns ( $\alpha$-amylase, $\alpha$-glucosidase, and pyranose oxidase(PROD)) was constructed for evaluation of the activity of the $\alpha$-amylase column. The immobilized $\alpha$-amylase column was applied to the apoenzyme reactivation method for detection of calcium(II) ions using the 


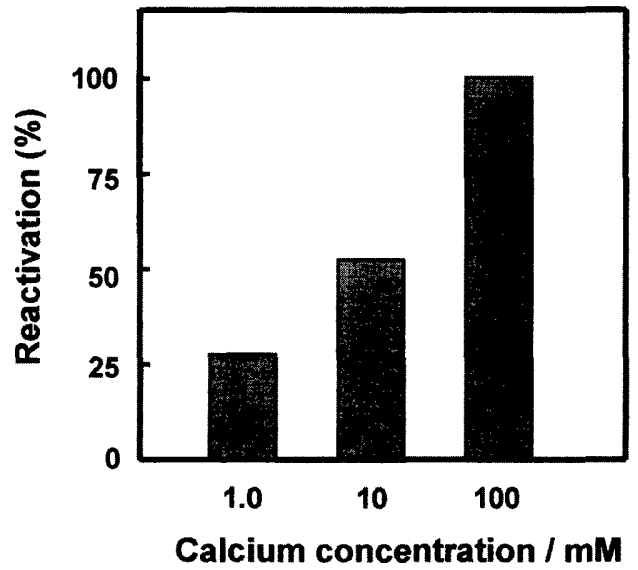

Fig. 5 Detection of calcium(II) ions based on the apoenzyme reactivation method using the $\alpha$-amylase column.

multi-enzyme columns. One millimolar calcium(II) ions solution was detectable by applying the apoenzyme reactivation method to the $\alpha$-amylase column as a recognition element. From the consideration of previous studies on the apoenzyme reactivation method applied to other enzymes such as determination of $\mathrm{Zn}$ (II) ions using alkaline phosphatase, the proposed method will be promising as a novel and powerful tool for determination of calcium (II) ions by further investigations.

\section{References}

1) I. Satoh, In Advances in Molecular and Cell Biology, 15 B, p.461, JAI Press Inc., Greenwich, CT, USA (1996).

2) I. Satoh and Y. Iijima, Sensors \& Actuators B, 24, 103 (1996).

3) I. Satoh and Y. Aoki, Denki Kagaku (presently Electrochemistry), 58, 1114 (1990).

4) I. Satoh, K. Ikeda, and N. Watanabe, Proc. $6^{\text {th }}$ Sensor Sym., p.203 (1986).

5) I. Satoh, T. Ariga and Y. Satoh, Proc. $4^{\text {th }}$ East Asian Conf. on Chemical Sensors, p.237 (1999).

6) I. Satoh, M. Kameyama, T. Ariga, and Y. Iida, Proc. 31st Chemical Sensor Sym., 16, Supplement B, p.40 (2000).

7) W. Heinen and A. M. Lauwers, Experientia Suppl., 26, 77 (1976).

8) I. Satoh and I. Sakurai, Ann. N.Y. Acad. Sci., 864, 493 (1998). 